\title{
Actor Critic Neural Network-Based Adaptive Control for MEMS Gyroscopes Suffering from Multiresource Disturbances
}

\author{
Chao Jing $(\mathbb{D}$ and Gangzhu Qiao \\ College of Data Science and Technology, North University of China, 3 Xueyuan Road, Taiyuan 030051, ShanXi, China \\ Correspondence should be addressed to Chao Jing; jingchao@nuc.edu.cn
}

Received 14 December 2020; Revised 23 December 2020; Accepted 5 July 2021; Published 30 September 2021

Academic Editor: Xingling Shao

Copyright (c) 2021 Chao Jing and Gangzhu Qiao. This is an open access article distributed under the Creative Commons Attribution License, which permits unrestricted use, distribution, and reproduction in any medium, provided the original work is properly cited.

In this paper, an actor critic neural network-based adaptive control scheme for micro-electro-mechanical system (MEMS) gyroscopes suffering from multiresource disturbances is proposed. Faced with multiresource interferences consisting of parametric uncertainties, strong couplings between axes, Coriolis forces, and variable external disturbances, an actor critic neural network is introduced, where the actor neural network is employed to estimate the packaged disturbances and the critic neural network is utilized to supervise the system performance. Hence, strong robustness against uncertainties and better tracking properties can be derived for MEMS gyroscopes. Aiming at handling the nonlinearities inherent in gyroscopes without analytically differentiating the virtual control signals, dynamic surface control (DSC) rather than backstepping control method is employed to divide the 2nd order system into two 1st order systems and design the actual control policy. Moreover, theoretical analyses along with simulation experiments are conducted with a view to validate the effectiveness of the proposed control approach.

\section{Introduction}

Owing to the microvolume, easy integration, and mass production, micro-electro-mechanical system (MEMS) gyroscopes are indispensable angular rate sensors in a myriad of areas including inertial guidance, aerospace, and national defense industry [1-5]. Typically, the accurate detection of angular rates is highly dependent on the high-precision control of drive axis and sense axis. However, MEMS gyroscopes are inherently accounted for strong uncertainties and nonlinearities, rendering the high-precision control a challenging issue.

Ideally, the parameters of MEMS gyroscopes are naturally fixed, including mass, damping parameters, and spring parameters, and mechanical motion of two axes are uncoupled. While in real word applications, manufacturing defects and unpredictable operation environments undesirably result in multiresource interferences, i.e., parametric uncertainties, mechanical coupling between axes, and external disturbances, which inevitably reduce the tracking accuracy of both axes and even lead to system instability.
Aiming at reducing the effects of uncertainties and enhancing system behaviours, several advanced schemes have been devised, such as neural networks (NNs) [6-8], extended state observers (ESOs) [9], and fuzzy logic systems (FLSs) [10], where the packaged disturbances are online estimated and compensated in the control policy. In [11], on the basis of universal approximation theorem, the RBFNN is employed to online estimate unknown system dynamics, effectively enhancing system robustness against uncertainties. In $[7-10,12]$, the tracking errors as well as estimation errors are bounded, while the tracking performance is usually neglected, which plays a central role in the highaccuracy measurement of angular rates. Hence, it is meaningful to devise a control scheme capable of recovering unknown interferences and supervising system tracking properties at the same time.

On the contrary, an appropriate stabilizing control policy is crucial to driving the output of MEMS gyroscopes to track the reference signals. In real-world applications, proportional integral derivative (PID) approaches $[13,14]$ are ubiquitous deployed to control nonlinear systems due to 
its theorical simplicity and easy implementation. However, in the existence of both system and external uncertainties, the PID-based methods cannot fulfil some high-accuracy tracking requirements, which is a fatal weakness of PID controllers. To simultaneously stabilize high-order nonlinear systems and compensate for the lumped disturbances, backstepping control schemes [15-18] are proposed, where the overall system is divided into several first-order systems and virtual control signals are developed to link up each subsystem. Nevertheless, the repeated analytical differentiations of virtual control signals may result in differentiating explosions, threating the normal operations of nonlinear systems. Hence, it is urgent and necessary to modify existing backstepping control approaches for MEMS gyroscopes without inducing such issues.

Oriented by the previous investigations, an actor critic NN-based adaptive control scheme for MEMS gyroscopes suffering from multiresource disturbances is proposed in this paper, the striking features of which can be summarized as follows:

Unlike the existing PID controller [13, 14], where the system uncertainties are neglected, the proposed control scheme can reduce the effects of lumped disturbances and stabilize the overall system. And, differing from previous backstepping control approaches [15-18] for MEMS gyroscopes that suffer from differentiating explosions, a low-pass filter is embedded to modify the controller design and avoid the analytical differentiations of virtual control signals.

Different from most NN or FLS-based control schemes [7-10,12], which can only ensure the ultimately uniformly bounded (UUB) properties of tracking errors, the proposed ACNN-based control scheme can simultaneously recover unknown dynamics and supervise tracking properties of MEMS gyroscope, resulting better robustness against uncertainties.

By resorting Lyapunov analyses, all the existing signals are proven to be UUB and a series of simulation verifications are presented to further validate the effectiveness of our proposed control approach.

\section{Problem Statements}

2.1. Kinetic Model of MEMS Gyroscopes. By resorting to [19-22], the kinetic model of MEMS gyroscopes is typically expressed as

$$
\begin{aligned}
m \ddot{x} & +d_{x x} \dot{x}+\left(d_{x y}-2 m \Omega_{Z}\right) \dot{y}+\left(k_{x x}-m \Omega_{Z}^{2}\right) x+k_{x y} y \\
& +\lambda_{x}=u_{x}, \\
m \ddot{y} & +d_{y y} \dot{y}+\left(d_{x y}+2 m \Omega_{Z}\right) \dot{x}+\left(k_{y y}-m \Omega_{Z}^{2}\right) y+k_{x y} x \\
& +\lambda_{y}=u_{y},
\end{aligned}
$$

where $m$ denotes the mass of internal moving mass, $\Omega_{Z}$ represents the rotation velocity, $x$ and $y$ are, respectively, the displacements of drive and sensitive modes, $k_{x x}$ and $k_{y y}$ represent linear spring terms, $d_{x x}$ and $d_{y y}$ refer to damping terms, $k_{x y}$ and $d_{x y}$ are the coupling coefficients, and $u_{x}$ and $u_{y}$ denote the input forces of drive and sensitive modes. Additionally, $\lambda_{x}$ and $\lambda_{y}$ describe the external disturbances. An overall structure of MEMS gyroscope is depicted in Figure 1.

Dividing both side of (1) by $m \omega_{0}^{2} l_{0}$, the following nondimensional model can be derived:

$$
\begin{aligned}
& \ddot{x}^{*}+\frac{d_{x x}}{m \omega_{0}} \dot{x}^{*}+\frac{\left(d_{x y}-2 m \Omega_{Z}\right)}{m \omega_{0}} \dot{y}^{*}+\frac{\left(k_{x x}-m \Omega_{Z}^{2}\right)}{m \omega_{0}^{2}} x^{*}+\frac{k_{x y}}{m \omega_{0}^{2}} y^{*} \\
& +\frac{\lambda_{x}}{m \omega_{0}^{2} l_{0}}=\frac{u_{x}}{m \omega_{0}^{2} l_{0}}, \\
& \ddot{y}^{*}+\frac{d_{y y}}{m \omega_{0}} \dot{y}^{*}+\frac{\left(d_{x y}+2 m \Omega_{Z}\right)}{m \omega_{0}} \dot{y}^{*}+\frac{\left(k_{y y}-m \Omega_{Z}^{2}\right)}{m \omega_{0}^{2}} y^{*}+\frac{k_{x y}}{m \omega_{0}^{2}} x^{*} \\
& +\frac{\lambda_{y}}{m \omega_{0}^{2} l_{0}}=\frac{u_{y}}{m \omega_{0}^{2} l_{0}},
\end{aligned}
$$

with

$$
\begin{aligned}
& \mathbf{x}_{p}=\left[\begin{array}{l}
x_{p 1} \\
x_{p 2}
\end{array}\right]=\left[\begin{array}{l}
x^{*} \\
y^{*}
\end{array}\right], \\
& \mathbf{x}_{v}=\left[\begin{array}{l}
x_{v 1} \\
x_{v 2}
\end{array}\right]=\left[\begin{array}{l}
\dot{x}^{*} \\
\dot{y}^{*}
\end{array}\right] \text {, } \\
& \mathbf{u}=\left[\begin{array}{l}
u_{1} \\
u_{2}
\end{array}\right]=\left[\begin{array}{c}
\frac{u_{x}}{m \omega_{0}^{2} l_{0}} \\
\frac{u_{y}}{m \omega_{0}^{2} l_{0}}
\end{array}\right], \\
& \boldsymbol{\Omega}=\left[\begin{array}{cc}
0 & \frac{\Omega_{Z}}{\omega_{0}} \\
-\frac{\Omega_{Z}}{\omega_{0}} & 0
\end{array}\right] \text {, } \\
& \mathbf{D}=\left[\begin{array}{ll}
\frac{d_{x x}}{m \omega_{0}} & \frac{d_{x y}}{m \omega_{0}} \\
\frac{d_{x y}}{m \omega_{0}} & \frac{d_{y y}}{m \omega_{0}}
\end{array}\right], \\
& \mathbf{K}=\left[\begin{array}{ll}
\frac{k_{x x}}{m \omega_{0}^{2}} & \frac{k_{x y}}{m \omega_{0}^{2}} \\
\frac{k_{x y}}{m \omega_{0}^{2}} & \frac{k_{y y}}{m \omega_{0}^{2}}
\end{array}\right] \text {, } \\
& \lambda=\left[\begin{array}{c}
\frac{\lambda_{x}}{m \omega_{0}^{2} l_{0}} \\
\frac{\lambda_{y}}{m \omega_{0}^{2} l_{0}}
\end{array}\right] .
\end{aligned}
$$




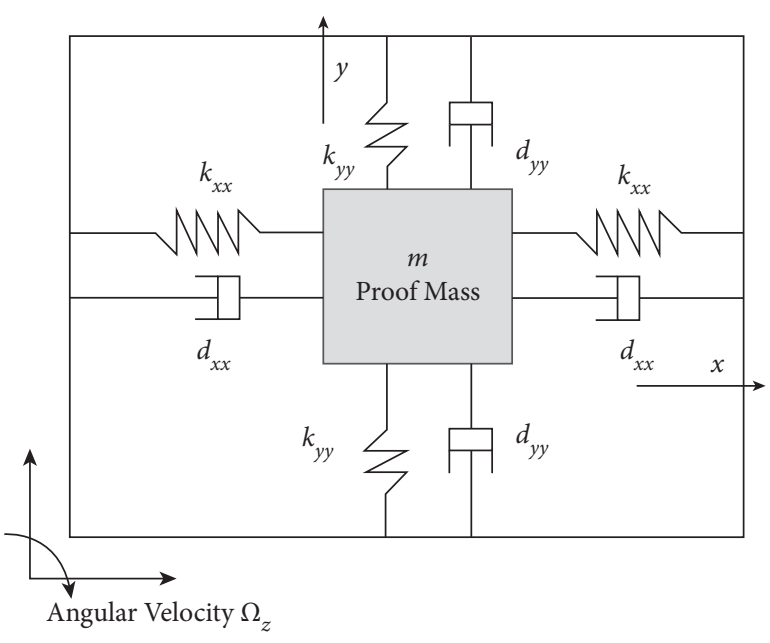

FIGURE 1: Overall structure of MEMS gyroscope.

In pursuit of description conciseness, (2) is further rewritten into the following strict-feedback vector form:

$$
\left\{\begin{array}{l}
\dot{\mathbf{x}}_{p}=\mathbf{x}_{v} \\
\dot{\mathbf{x}}_{v}=\mathbf{u}+\mathbf{F}
\end{array}\right.
$$

with $\mathbf{F}=\left[F_{1}, F_{2}\right]^{\mathrm{T}}=-(\mathbf{D}-2 \Omega) \mathbf{x}_{v}-\left(\mathbf{K}-\Omega^{2}\right) \mathbf{x}_{p}-\lambda$ defined as the packaged disturbances.

2.2. Control Objective. Our objective is to devise a control policy $\mathbf{u}$ based on ACNNs and DSC schemes, which is capable of driving the output states of MEMS gyroscopes $\mathbf{x}_{p}$ to track the reference signal $\mathbf{y}_{r}=\left[y_{r 1}, y_{r 2}\right]^{\mathrm{T}}$ as accurately as possible, in the existence of multisource interferences, i.e., $\mathbf{F}$.

\section{Main Results}

3.1. AC Neural Networks. In what follows, the actor neural network is employed to reconstruct the packaged disturbance $\mathbf{F}$, which is formulated as

$$
F_{i}(\bar{x})=\mathbf{W}_{a i} \boldsymbol{\theta}_{a i}(\bar{x})+\delta_{a i}(\bar{x}) .
$$

where $\mathbf{W}_{a i}=\left[W_{a i, 1}, W_{a i, 2}, \ldots, W_{a i, l}\right]^{\mathrm{T}} \in \mathbf{R}^{l}$ denotes the weight vector and $\delta_{a i}(\bar{x})$ describes the reconstruction errors satisfying $\delta_{a i}(\bar{x}) \leq \bar{\delta}_{a i}$ with $\bar{\delta}_{a i}$ being a positive finite constant. Define that $\widehat{W}_{a i}=\left[\widehat{W}_{a i, 1}, \widehat{W}_{a i, 2}, \ldots, \widehat{W}_{a i, l}\right]^{T} \in \mathbf{R}^{l}$ is estimation of $\mathbf{W}_{a i}$ and $\widetilde{W}_{a i}=\widehat{W}_{a i}-\mathbf{W}_{a i}$ denotes the estimation error. $\theta_{a i}(\bar{x})$ is the active function vector, which is chosen as the commonly used sigmoid function.

Herein, in order to supervise the tracking properties in real time, a reinforcement function is devised in the following form similar to [23]

$$
S_{i}=\frac{\bar{S}_{i}}{1+\exp \left(-\tau_{i} e_{p i}\right)}-\frac{\bar{S}_{i}}{1+\exp \left(\tau_{i} e_{p i}\right)},
$$

where $\tau_{i}$ and $\bar{S}_{i}$ are positive design parameters.

Subsequently, the critic function is defined as

$$
S_{c i}=\varphi_{i} S_{i}+\varphi_{i} S_{i} \mathbf{W}_{c i} \boldsymbol{\theta}_{c i}(\bar{x}),
$$

where $\quad \mathbf{W}_{c i}=\left[W_{c i, 1}, W_{c i, 2}, \ldots, W_{c i, l}\right]^{T} \in \mathbf{R}^{l}$ denotes the weight vector. Define that $\widehat{W}_{c i}=\left[\widehat{W}_{c i, 1}, \widehat{W}_{c i, 2}, \ldots, \widehat{W}_{c i, l}\right]^{T}$ $\in \mathbf{R}^{l}$ is estimation of $\mathbf{W}_{c i}$ and $\widetilde{W}_{c i}=\widehat{W}_{c i}-\mathbf{W}_{c i}$ denotes the estimation error. $\theta_{c i}(\bar{x})$ is the active function vector, which can be calculated in the following form:

Apparently, $S_{i}$ is always bounded within $\left[-\bar{S}_{i}, \bar{S}_{i}\right]$, and the smaller $\left|e_{p i}\right|$ is, the closer $S_{i}$ is to zero. Hence, the control objective of driving $\mathbf{x}_{p}$ to track $\mathbf{y}_{r}$ boils down to ensuring that $S_{c}$ is as close to zero as possible. The actual critic signal is formulated as follows:

$$
\widehat{S}_{c i}=\varphi_{i} S_{i}+\left\|\varphi_{i} S_{i}\right\| \widehat{W}_{c i} \boldsymbol{\theta}_{c i}(\bar{x})
$$

where $\widehat{W}_{c i}$ is the estimation of $\mathbf{W}_{c i}$.

3.2. Controller Design. The following error dynamics are defined in advance, aiming at promoting the controller design and stability analysis:

$$
\begin{gathered}
e_{p i} \triangleq x_{p i}-y_{r i}, \\
e_{v i} \triangleq x_{v i}-\bar{\nu}_{i}, \\
\varphi_{i} \triangleq \bar{v}_{i}-v_{i},
\end{gathered}
$$

with $\nu_{i}$ being the virtual control policy and $\bar{\nu}_{i}$ is its low-pass filtered signal, which are formulated as

$$
\begin{aligned}
\chi_{i} \dot{\bar{\nu}}_{i}+\bar{\nu}_{i} & =v_{i}, \\
v_{i}(0) & =\bar{\nu}_{i}(0),
\end{aligned}
$$

with $\chi_{i}$ being a positive time-delay parameter.

Taking the time derivative of $\varphi_{i} \triangleq \bar{\nu}_{i}-v_{i}$ and utilizing (10), the following equation can be guaranteed:

$$
\dot{\varphi}_{i}+\frac{\varphi_{i}}{\chi_{i}}=-\dot{v}_{i}
$$

Note that $\dot{v}_{i}$ is a continuous and bounded function satisfying $\left|\dot{v}_{i}\right| \leq \nu_{0}$ and $v_{0}$ is a positive constant.

Step 1. at this point, devise the Lyapunov candidate of displacement loop as $V_{p}=\sum_{i=1}^{2}\left(e_{p i}^{2}+\varphi_{i}^{2}\right) / 2$. Differentiating it along with time and recalling the kinetic model of MEMS gyroscope (4), one has

$$
\begin{aligned}
\dot{V}_{p} & =\sum_{i=1}^{2}\left(\dot{e}_{p i} e_{p i}+\dot{\varphi}_{i} \varphi_{i}\right) \\
& =\sum_{i=1}^{2}\left(\left(e_{v i}+v_{i}+\varphi_{i}-\dot{y}_{r i}\right) e_{p i}+\dot{\varphi}_{i} \varphi_{i}\right) .
\end{aligned}
$$

In order to stabilize $V_{p}$, the virtual control policy is developed as

$$
v_{i}=-k_{p i} e_{p i}+\dot{y}_{r i} .
$$

According to Young's inequality, the following inequalities hold: 


$$
e_{v i} e_{p i} \leq \frac{1}{2} e_{v i}^{2}+\frac{1}{2} e_{p i}^{2}, \varphi_{i} e_{p i} \leq \frac{1}{2} \varphi_{i}^{2}+\frac{1}{2} e_{p i}^{2}, \varphi_{i} \dot{v}_{i} \leq \frac{1}{2} \varphi_{i}^{2}+\frac{1}{2} v_{0}^{2}
$$

Subsequently, inserting (13) and (14) into (12), it follows that

$$
\dot{V}_{p} \leq \sum_{i=1}^{2}\left(-k_{p i} e_{p i}^{2}+\frac{1}{2} e_{v i}^{2}+e_{p i}^{2}+\varphi_{i}^{2}-\frac{1}{\chi_{i}} \varphi_{i}^{2}+\frac{1}{2} v_{0}^{2}\right)
$$

Step 2. construct the Lyapunov candidate of velocity loop as $V_{v}=\sum_{i=1}^{2}\left(\bar{S}_{i}\left(\ln \left(1+\exp \left(-\tau_{i} e_{p i}\right)\right)+\ln \left(1+\exp \left(\tau_{i} e_{p i}\right)\right)\right) / \tau_{i}+\right.$ $\left.\dot{\tilde{W}}_{a i}^{T} \widetilde{W}_{a i} / 2 \sigma_{a i}+\dot{\tilde{W}}_{c i}^{T} \widetilde{W}_{c i} / 2 \sigma_{c i}\right)$. Differentiating it along with time and recollecting the kinetic dynamics of MEMS gyroscope (4), it follows

$$
\begin{aligned}
\dot{V}_{i} & =\sum_{i=1}^{2}\left(S_{i} \dot{e}_{v i}+\frac{\dot{\tilde{W}}_{a i}^{T} \tilde{W}_{a i}}{\sigma_{a i}}+\frac{\dot{\tilde{W}}_{c i}^{T} \tilde{W}_{c i}}{\sigma_{c i}}\right), \\
& =\sum_{i=1}^{2}\left(S_{i}\left(u_{i}+F_{i}-\dot{\bar{v}}_{i}\right)+\frac{\dot{\tilde{W}}_{a i}^{T} \tilde{W}_{a i}}{\sigma_{a i}}+\frac{\dot{\tilde{W}}_{c i}^{T} \tilde{W}_{c i}}{\sigma_{c i}}\right) .
\end{aligned}
$$

In order to stabilize the velocity loop and supervise the tracking performance in real time for MEMS gyroscopes, the actual control policy as well as adaptive updating policy are presented in (18)-(20):

$$
\begin{aligned}
u_{i} & =-k_{v i} S_{i}-\widehat{W}_{a i} \boldsymbol{\theta}_{a i}+\dot{\bar{\nu}}_{i}, \\
\dot{\hat{W}}_{a i}^{T} & =\sigma_{a i}\left(\widehat{S}_{c i} \boldsymbol{\theta}_{a i}^{T}-\kappa_{a i} \widehat{W}_{a i}^{T}\right), \\
\dot{\hat{W}}_{c i}^{T} & =-\sigma_{c i}\left(\varphi_{i} S_{i} \boldsymbol{\theta}_{c i}^{T}\left(\widehat{W}_{a i} \boldsymbol{\theta}_{a i}\right)^{T}+\kappa_{c i} \widehat{W}_{c i}^{T}\right),
\end{aligned}
$$

with $u_{m i}$ being the modification control term, which can be derived from (19) and (20). $\sigma_{a i}, \sigma_{c i}, \kappa_{a i}$, and $\kappa_{c i}$ are commonly positive constants.

Via inserting (17)-(19) into (16) and recalling (5) as well as (7), the following inequality can be ensured:

$$
\begin{aligned}
\dot{V}_{i} & =\sum_{i=1}^{2}\left(\left(-\widetilde{W}_{a i} \boldsymbol{\theta}_{a i}+\delta_{a i}-k_{v i} S_{i}\right) \varphi_{i} S_{i}-k_{v i} S_{i}^{2}+\left(\widehat{S}_{c i} \boldsymbol{\theta}_{a i}^{T}-\kappa_{a i} \widehat{W}_{a i}^{T}\right) \widetilde{W}_{a i}-\left(\varphi_{i} S_{i} \boldsymbol{\theta}_{c i}^{T}\left(\widehat{W}_{a i} \boldsymbol{\theta}_{a i}\right)^{T}+\kappa_{c i} \widehat{W}_{c i}^{T}\right) \widetilde{W}_{c i}\right) \\
& =\sum_{i=1}^{2}\left(\left(-\widetilde{W}_{a i} \boldsymbol{\theta}_{a i}+\delta_{a i}-k_{v i} S_{i}\right) \varphi_{i} S_{i}-k_{v i} S_{i}^{2}-\kappa_{a i} \widehat{W}_{a i}^{T} \widetilde{W}_{a i}+\left(\varphi_{i} S_{i}+\varphi_{i} S_{i} \widehat{W}_{c i} \boldsymbol{\theta}_{c i}\right) \boldsymbol{\theta}_{a i}^{T} \tilde{W}_{a i}-\kappa_{c i} \widehat{W}_{c i}^{T} \widetilde{W}_{c i}-\left(\varphi_{i} S_{i} \boldsymbol{\theta}_{c i}^{T}\right) \widetilde{W}_{c i}\left(\widehat{W}_{a i} \boldsymbol{\theta}_{a i}\right)^{T}\right)
\end{aligned}
$$

\section{Stability Analyses}

Herein, the main theorem of our paper is presented as, on the basis of the virtual control policy, the actual control policy and the adaptive updating policy; all the error dynamics involved in the closed-loop MEMS gyroscope system are UUB.
Proof. In the first place, constructing the general Lyapunov function as $V=V_{p}+V_{v}$, differentiating it along with time and employing (15), (20), it can be derived that

$$
\begin{aligned}
\dot{V}= & \dot{V}_{p}+\dot{V}_{v} \leq \sum_{i=1}^{2}\left(-k_{p i} e_{p i}^{2}+\frac{1}{2} e_{v i}^{2}+e_{p i}^{2}+\varphi_{i}^{2}-\frac{1}{\chi_{i}} \varphi_{i}^{2}+\frac{1}{2} v_{0}^{2}\right)+\sum_{i=1}^{2}\left(\left(-\widetilde{W}_{a i} \boldsymbol{\theta}_{a i}+\delta_{a i}-u_{m i}\right) \varphi_{i} S_{i}-k_{v i} S_{i}^{2}-\kappa_{a i} \widehat{W}_{a i}^{T} \widetilde{W}_{a i}\right. \\
& \left.+\left(\varphi_{i} S_{i}+\varphi_{i} S_{i} \widehat{W}_{c i} \boldsymbol{\theta}_{c i}\right) \boldsymbol{\theta}_{a i}^{T} \tilde{W}_{a i}-\kappa_{c i} \widehat{W}_{c i}^{T} \tilde{W}_{c i}-\left(\varphi_{i} S_{i} \boldsymbol{\theta}_{c i}^{T}\right) \tilde{W}_{c i}\left(\widehat{W}_{a i} \boldsymbol{\theta}_{a i}\right)^{T}\right) .
\end{aligned}
$$

Due to Young's inequality and the fact that $\left\|\theta_{c i}\right\| \leq \bar{\theta}_{c i}$, the following inequalities hold: 
TABLE 1: Design parameters of this simulation.

\begin{tabular}{ll}
\hline Section & \multicolumn{1}{c}{ Parameters } \\
\hline \multirow{2}{*}{ ACNN } & $\sigma_{a 1}=1200, \sigma_{a 2}=1100, \kappa_{a 1}=15, \kappa_{a 2}=12$ \\
& $\sigma_{c 1}=670, \sigma_{c 2}=840, \kappa_{c 1}=8, \kappa_{c 2}=7, \tau_{1}=\tau_{2}=0.5$ \\
\hline Controller & $k_{p 1}=5, k_{p 2}=5, \chi_{1}=\chi_{2}=0.05, k_{v 1}=10, k_{v 2}=10$ \\
\hline
\end{tabular}
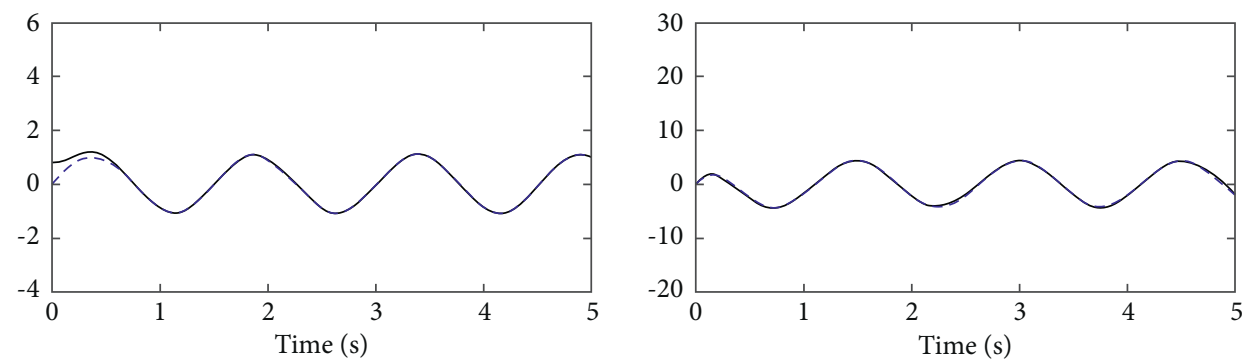

$$
\begin{aligned}
& --x_{p 1} \\
& -y_{r 1}
\end{aligned}
$$

$-\cdots x_{v 1}$
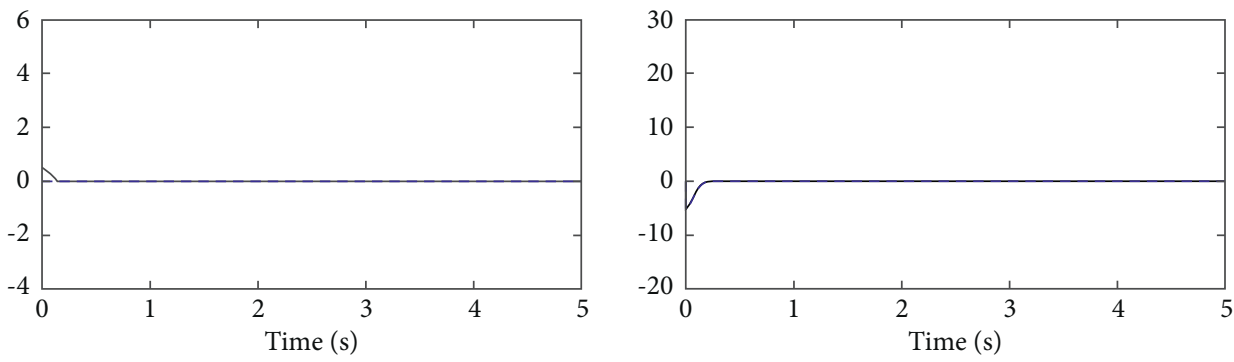

$--x_{p 2}$
$-y_{r 2}$

$-x_{v 2}$

FIgURE 2: Tracking performance of both displacement and velocity loops.

$$
\begin{aligned}
\left(-\widetilde{W}_{a i} \boldsymbol{\theta}_{a i}+\delta_{a i}\right) \varphi_{i} S_{i} & +\left(\varphi_{i} S_{i}+\varphi_{i} S_{i} \widehat{W}_{c i} \boldsymbol{\theta}_{c i}\right) \boldsymbol{\theta}_{a i}^{T} \tilde{W}_{a i}-\varphi_{i} S_{i} \boldsymbol{\theta}_{c i}^{T} \tilde{W}_{c i}\left(\widehat{W}_{a i} \boldsymbol{\theta}_{a i}\right)^{T} \leq \varphi_{i} S_{i}\left(\left\|\widehat{W}_{a i}\right\| \bar{\theta}_{a i} \bar{\theta}_{c i}\left\|\mathbf{W}_{c i}\right\|+\left\|\mathbf{W}_{a i}^{T}\right\| \bar{\theta}_{a i} \bar{\theta}_{c i}\left\|\widehat{W}_{c i}\right\|+\bar{\delta}_{a i}\right) \\
& -\widehat{W}_{c i}^{T} \tilde{W}_{c i} \leq \frac{1}{2}\left(\left\|\mathbf{W}_{c i}\right\|^{2}-\left\|\widetilde{W}_{c i}\right\|^{2}\right),-\widehat{W}_{a i}^{T} \tilde{W}_{a i} \leq \frac{1}{2}\left(\left\|\mathbf{W}_{a i}\right\|^{2}-\left\|\widetilde{W}_{a i}\right\|^{2}\right)
\end{aligned}
$$

Hence, (21) can be further rewritten as

$$
\dot{V} \leq \sum_{i=1}^{2}\left(-\left(k_{p i}-1\right) e_{p i}^{2}-\left(\frac{1}{\chi_{i}}-1\right) \varphi_{i}^{2}-k_{v i} S_{i}^{2}-\frac{1}{2} \kappa_{c i}\left\|\tilde{W}_{c i}\right\|^{2}-\frac{1}{2} \kappa_{a i}\left\|\tilde{W}_{a i}\right\|^{2}+\delta_{a i} \varphi_{i} S_{i}+\varphi_{i} S_{i} G_{i}+\frac{1}{2} v_{0}^{2}+\frac{1}{2}\left\|\mathbf{W}_{c i}\right\|\left\|^{2}+\frac{1}{2}\right\| \mathbf{W}_{a i} \|^{2}\right),
$$

with $G_{i}=\left\|\widehat{W}_{a i}\right\| \bar{\theta}_{a i} \bar{\theta}_{c i}\left\|\mathbf{W}_{c i}\right\|+\left\|\mathbf{W}_{a i}^{T}\right\| \bar{\theta}_{a i} \bar{\theta}_{c i}\left\|\widehat{W}_{c i}\right\|+\bar{\delta}_{a i}$.

Moreover, employing Young's inequality, it can be achieved that

$$
\delta_{a i} \varphi_{i} S_{i} \leq \frac{1}{2} \varphi_{i} S_{i}^{2}+\frac{1}{2} \varphi_{i} \bar{\delta}_{a i}^{2}, \varphi_{i} S_{i} G_{i} \leq \frac{1}{2} \varphi_{i} S_{i}^{2}+\frac{1}{2} \varphi_{i} G_{i}^{2} .
$$

Substituting (24) into (23) further produces

$$
\dot{V} \leq \sum_{i=1}^{2}\left(-\left(k_{p i}-1\right) e_{p i}^{2}-\left(\frac{1}{\chi_{i}}-1\right) \varphi_{i}^{2}-\left(k_{v i}-1\right) S_{i}^{2}-\frac{1}{2} \kappa_{c i}\left\|\widetilde{W}_{c i}\right\|^{2}-\frac{1}{2} \kappa_{a i}\left\|\widetilde{W}_{a i}\right\|^{2}+\frac{1}{2} v_{0}^{2}+\frac{1}{2}\left\|\mathbf{W}_{c i}\right\|^{2}+\frac{1}{2}\left\|\mathbf{W}_{a i}\right\|^{2}+\frac{1}{2} \varphi_{i} \bar{\delta}_{a i}^{2}+\frac{1}{2} \varphi_{i} G_{i}^{2}\right) .
$$


From (25), it can be easily discovered that, as long as $\left(k_{p i}-1\right)>p,\left(1 / \chi_{i}-1\right)>p,\left(k_{v i}-1\right)>p, \kappa_{c i} / 2>p, \kappa_{a i} / 2>p$ and $\Gamma / V(0)<p$ hold with $p$ being a positive constant and $\Gamma=v_{0}^{2} / 2+\left\|\mathbf{W}_{c i}\right\|^{2} / 2+1 / 2\left\|\mathbf{W}_{a i}\right\|^{2} / 2+\varphi_{i} \bar{\delta}_{a i}^{2} / 2+\varphi_{i} G_{i}^{2} / 2, \quad \dot{V} \leq 0$ always holds for $\forall t \geq 0$. Hence, all the error signals considered in $V$ are proven to be $\mathrm{UUB}$, and the proof is completed.
To verify the practicability of the proposed control scheme, a series of simulations are presented for MEMS gyroscopes in the proceeding section. By resorting to [20], the physical parameters of MEMS gyroscopes are typically chosen as

$$
\begin{aligned}
m & =1.8 \times 10^{-7} \mathrm{~kg}, k_{x x}=63.955 \mathrm{~N} / \mathrm{m}, k_{y y}=95.92 \mathrm{~N} / \mathrm{m}, k_{x y}=12.779 \mathrm{~N} / \mathrm{m}, \\
d_{x x} & =1.8 \times 10^{-6} \mathrm{Ns} / \mathrm{m}, d_{y y}=1.8 \times 10^{-6} \mathrm{Ns} / \mathrm{m}, d_{x y}=3.6 \times 10^{-7} \mathrm{Ns} / \mathrm{m}, k_{x}^{2}=355.3, \\
k_{y}^{2} & =355.3, k_{x y}=70.99, D_{x}=0.01, D_{y}=0.01, D_{x y}=0.002, \Omega_{z}=0.1 .
\end{aligned}
$$

And, the following time-varying functions are utilized to simulate the unknown external disturbances:

$$
\lambda(t)=\left[\begin{array}{c}
6 \cos (3 t)+6 \sin (2 t) \exp (-t)+3 \\
3 \cos (3 t) \exp (-0.5 t)+4 \sin (2 t)-2
\end{array}\right], \quad t \geq 0
$$

Define the reference signal as $\mathbf{y}_{r}=[\sin (4.17 t), 0,]^{T}$. All the design parameters existing in the proposed control method are given in Table 1.

The tracking performance of displacement loop and velocity loop is presented in Figure 2, where satisfactory tracking accuracy can be discovered, which can be attributed to the effectiveness of DSC approach. Even in the presence of unknown dynamics, the tracking performance is rarely degraded, mainly due to the implementation of ACNN, which is able to recover system uncertainties and supervise system tracking performance at the same time.

Herein, it is notable that the issue of differentiation explosion is effectively avoided, mainly owing to the filtered signal is involved in the velocity loop controller design rather than the analytically differentiated signals. Moreover, although, in the existence of unknown multiresource interferences, the tracking performance is not degraded severely by resorting to the employed ACNN scheme and unlike most existing control schemes based on NN or FLS [7-10,12], which can only ensure the UUB behaviours of tracking errors, the proposed ACNN-based control scheme can recover unknown dynamics and supervise tracking properties of MEMS gyroscope at the same time, validating the robustness of our algorithm against uncertainties.

\section{Conclusions}

In this paper, an actor critic neural network-based adaptive control scheme for MEMS gyroscopes subject to multiresource disturbances is proposed. By combining an actor $\mathrm{NN}$ with a critic NN, the packaged disturbances can be effectively suppressed and system performance can be supervised at the same time, such that better tracking properties and stronger robustness against unknown dynamics can be guaranteed. Through inserting a first-order filter between the displacement and velocity loops of MEMS gyroscope, a DSC controller is constructed to ensure the stabilization for both displacement and velocity loops and avoid unexpected differentiation explosion. Moreover, all system dynamics are proven to be UUB via Lyapunov synthesis and a series of simulations through MATLAB/ SIMULINK are presented in the end of this paper, validating the effectiveness of our algorithm. In the near future, we may modify the ACNN by combining the fuzzy logics with neural networks [24, 25], aiming at further enhancing its generalization ability. At the same time, prescribed performance of tracking error will be considered for MEMS gyroscope to improve transient and steady-state tracking accuracy.

\section{Data Availability}

The data used to support the findings of the study are available from the corresponding author upon request.

\section{Conflicts of Interest}

The authors declare that they have no conflicts of interest.

\section{References}

[1] M. C. Bingol and O. Aydogmus, "Performing predefined tasks using the human-robot interaction on speech recognition for an industrial robot," Engineering Applications of Artificial Intelligence, vol. 95, Article ID 103903, 2020.

[2] Y. Cao, J. Figurero, W. Li, Z. Chen, Z. L. Wang, and N. Sepúlveda, "Understanding the dynamic response in ferroelectret nanogenerators to enable self-powered tactile systems and human-controlled micro-robots," Nanomaterials and Energy, vol. 63, Article ID 103852, 2019.

[3] M. C. Bingol and O. Aydogmus, "Performing predefined tasks using the human-robot interaction on speech recognition for an industrial robot," Engineering Applications of Artificial Intelligence, vol. 95, Article ID 103903, 2019.

[4] G. Wu, B. Han, D. D. Cheam et al., "Development of sixdegree-of-freedom inertial sensors with an 8-in advanced MEMS fabrication platform," IEEE Transactions on Industrial Electronics, vol. 66, no. 5, pp. 3835-3842, 2019.

[5] Y. Ren, X. Chen, Y. Cai, H. Zhang, C. Xin, and Q. Liu, "Attitude-rate measurement and control integration using magnetically suspended control and sensitive gyroscopes," 
IEEE Transactions on Industrial Electronics, vol. 65, no. 6, pp. 4921-4932, 2018.

[6] J. Fei and C. Lu, "Adaptive fractional order sliding mode controller with neural estimator," Journal of the Franklin Institute, vol. 355, no. 5, pp. 2369-2391, 2018.

[7] J. Fei and W. Yan, "Adaptive global fast terminal sliding mode control of MEMS gyroscope using fuzzy-neural-network," in Proceedings of the 33rd Chinese Control Conference, 2014, pp. 15-20, Nanjing, China, July 2014.

[8] X. Shao, Y. Shi, W. Zhang, and H. Cao, "Neurodynamic approximation-based quantized control with improved transient performances for microelectromechanical system gyroscopes: theory and experimental results," IEEE Transactions on Industrial Electronics, vol. 68, no. 10, pp. 99729983, 2021.

[9] X. Shao, J. Liu, H. Cao, C. Shen, and H. Wang, "Robust dynamic surface trajectory tracking control for a quadrotor UAV via extended state observer," International Journal of Robust and Nonlinear Control, vol. 28, no. 7, pp. 2700-2719, 2018.

[10] X. Shao, H. Si, and W. Zhang, "Fuzzy wavelet neural control with improved prescribed performance for MEMS gyroscope subject to input quantization," Fuzzy Sets and Systems, vol. 411, pp. 136-154, 2021.

[11] X. Shao, Z. Cao, and H. Si, "Neurodynamic formation maneuvering control with modified prescribed performances for networked uncertain quadrotors," IEEE Systems Journal, vol. 2020, Article ID 3022901, 12 pages, 2020.

[12] X. Shao, Y. Shi, and W. Zhang, "Fault-tolerant quantized control for flexible air-breathing hypersonic vehicles with appointed-time tracking performances," IEEE Transactions on Aerospace and Electronic Systems, vol. 57, no. 2, pp. 12611273, 2021.

[13] S. Sungsu Park and R. Horowitz, "Adaptive control for the conventional mode of operation of MEMS gyroscopes," Journal of Microelectromechanical Systems, vol. 12, no. 1, pp. 101-108, 2003.

[14] N.-C. Tsai and C.-Y. Sue, "Integrated model reference adaptive control and time-varying angular rate estimation for micro-machined gyroscopes," International Journal of Control, vol. 83, no. 2, pp. 246-256, 2010.

[15] J. Peng and R. Dubay, "Adaptive fuzzy backstepping control for a class of uncertain nonlinear strict-feedback systems based on dynamic surface control approach," Expert Systems with Applications, vol. 120, pp. 239-252, 2019.

[16] T. P. Zhang and S. S. Ge, "Adaptive dynamic surface control of nonlinear systems with unknown dead zone in pure feedback form," Automatica, vol. 44, no. 7, pp. 1895-1903, 2008.

[17] T. S. Tie-Shan Li, D. Dan Wang, G. Gang Feng, and S. C. ShaoCheng Tong, "A DSC approach to robust adaptive NN tracking control for strict-feedback nonlinear systems," IEEE Transactions on Systems, Man, and Cybernetics, Part B (Cybernetics), vol. 40, no. 3, pp. 915-927, 2010.

[18] Y. Pan and H. Yu, "Dynamic surface control via singular perturbation analysis," Automatica, vol. 57, pp. 39-33, 2015.

[19] N. Euler-Rolle, I. Škrjanc, C. Hametner, and S. Jakubek, "Automated generation of feedforward control using feedback linearization of local model networks," Engineering Applications of Artificial Intelligence, vol. 50, pp. 320-330, 2016.

[20] H. Si, X. Shao, and W. Zhang, "MLP-based neural guaranteed performance control for MEMS gyroscope with logarithmic quantizer," IEEE Access, vol. 8, pp. 38596-38605, 2020.
[21] M. Marx, S. Rombach, S. Nessler, D. De Dorigo, and Y. Manoli, "A 141- \$ \mu\$ W high-voltage MEMS gyroscope drive interface circuit based on flying capacitors," IEEE Journal of Solid-State Circuits, vol. 54, no. 2, pp. 511-523, 2019.

[22] Y. Shi, X. L. Shao, and W. D. Zhang, "Neural observer-based quantized output feedback control for MEMS gyroscopes with guaranteed transient performance," Aerospace Science and Technology, vol. 105, Article ID 106055, 2020.

[23] X. Bu, Y. Xiao, and H. Lei, "An adaptive critic design-based fuzzy neural controller for hypersonic vehicles: predefined behavioral nonaffine control," IEEE, vol. 24, no. 4, pp. 1871-1881, 2019.

[24] X. Bu, "Air-breathing hypersonic vehicles funnel control using neural approximation of non-affine dynamics," IEEE, vol. 23, no. 5, pp. 2099-2108, 2018.

[25] X. Bu and Q. Qi, "Fuzzy optimal tracking control of hypersonic flight vehicles via single-network adaptive critic design," IEEE Transactions on Fuzzy Systems, vol. 2020, Article ID 3036706, 1 page, 2020. 